\title{
ANT BASED ROUTING ALGORITHM OVER CLUSTERING TECHNIQUE TO PROVIDE BETTER COMMUNICATION FOR MANETS WITH MINIMUM HOP COUNT
}

\author{
Sowmya $\mathrm{K} \mathrm{S}^{1}$ and Cauvery $\mathrm{N} \mathrm{K}^{2}$
}

\begin{abstract}
Communication is the basic requirement for the recent trends of technology. Infrastructure provision cannot be fulfilled according to the requirement due to lot of man power and materials involved. Also if one node wants to communicate to multiple nodes, then multicasting is a challenge. Now the trend is to create network where ever you are or easily be a part of network which is existing. In this paper this can be achieved in Mobile Ad hoc Networks (MANETs) by creating clusters and election of Cluster Leader (CL) with the help of Weighted Clustering Algorithm (WCA). Cluster leaders of different clusters will communicate through a technique under Swarm Intelligence called Ant Colony Optimization (ACO). ACO uses Ant Based Route Discovery (ABRD) with the help of Forward Ant (FANT) and Backwards Ant (BANT). Once the path between the CL is identified through the pheromone deposited by FANT and BANT, it can be used to multicast data. Simulations on NS-2 show that the communication from one mobile node to any other mobile node can be achieved in just four hops. Multicasting can be achieved with less packet loss.
\end{abstract}

Keywords-MANETs, ACO, Clustering, WCA, Multicasting, Route discovery

\section{INTRODUCTION}

If mobile nodes are densely populated then it is very difficult for a mobile node to identify the destination node [1]. In flat routing technique, Route discovery process consumes lot of energy and route request packet occupies lot of bandwidth. All the intermediate nodes are expected to contribute the route discovery process by giving the information regarding their neighbors. Also fill their routing table with the information they collect from these route discovery packet and route reply packet. Flat routing technique consumes lot of power and delay is increased. Hence hierarchical method has to be implemented to overcome the above mentioned drawbacks [2].

Power aware routing can be implemented to reduce the power consumed in route discovery process. The amount of data stored on each of these neighboring nodes can also be reduced. As less number of nodes is involved in forwarding the packets on the wireless medium bandwidth will also be less consumed [3].

Multicasting is a technique where one mobile node can communicate to multiple mobile nodes. Here there is a single source and multiple destinations scattered across the MANET [4]. Multicasting on MANETs can be achieved by the MAODV. In MAODV, all the nodes willing

\footnotetext{
${ }^{1}$ Dept of ISE, BMSCE, Bangalore, India

${ }^{2}$ Dept of ISE, RVCE, Bangalore, India
} 
to join multicast tree will form a spanning tree and then the data can be communicated in one to many fashion. But the amount of packet loss will be more in MAODV.

\section{BASICS AND BACKGROUND}

\section{A. Clustering Technique}

Clustering technique can be followed to achieve power aware routing in MANETs. In clustering technique, nodes which are geographically close together form clusters. If stability of clusters is not maintained then they may cause rapid failure [5]. After forming cluster, a CL will be elected to handle the communication within the cluster. CL election is done in following ways [6].

Lowest id clustering algorithm: In this algorithm, each node in the cluster is given a unique id and the cluster leader is the one with lowest id. Notable disadvantage of this system is that the resources of the cluster leader will be drained out at the higher rate.

Highest degree clustering algorithm: Degree will be assigned to each node based on its connectivity and the routing table entries. Node with highest degree will be considered as a cluster leader.

K-hop connectivity algorithm: In this algorithm, lowest id and highest degree is merged. A node with highest degree will be considered as a cluster leader. In case of tie between the degrees of two nodes, then the node with lowest id will become the cluster leader.

Weighted Clustering Algorithm (WCA): In this algorithm, weights will be assigned to the factors like mobility of a node $(\mathrm{Mn})$, power available $(\mathrm{P})$, transmission rate $(\mathrm{Tr})$ and the power consumed for transmission (Tp).

Weight of a node $(\mathrm{Wn})$ is calculated using a formula

$\mathrm{Wn}=\mathrm{w} 1 * \mathrm{Mn}+\mathrm{w} 2 * \mathrm{P}+\mathrm{w} 3 * \mathrm{Tr}+\mathrm{w} 4 * \mathrm{Tp}$

A node with highest weight will be considered as a cluster leader.

B. Ant Colony Optimization:

Interaction between cluster leaders in MANETs usually will happen through a well-known routing algorithm called Ad hoc On demand Distance Vector (AODV). In AODV, Route Request Packet (RREQ) will travel all the way from source node to the destination node passing through the nodes coming on the way. These RREQ will be used to update the routing tables of the intermediate mobile node thus by wasting their resources. And a Route Reply (RREP) will come again on all possible ways to the source node to indicate that the destination is now aware and ready to receive the messages.

A technique under swarm intelligence called ACO can be adapted to reduce the number of participants in the route discovery phase [7]. ACO over clustering technique will further reduce delay.

ACO uses ABRD where algorithm resembles the food searching mechanism adapted by ants. These ants deposit a chemical called pheromone on the nodes it visits. Source is considered as ant colony and the destination is considered as food [8].

From the source, Forward Ant (FANT) will be sent to find the destination by depositing the pheromone. Destination will construct Backward Ant (BANT) and send it to the source over the path constructed by the pheromone deposited. Each time ant visit the path formed by pheromone, the pheromone value will be incremented indicating that the path is being reused. Here pheromones are used to maintain the path rather than updating the routing table with various values.

Pseudo Code of the ACO algorithm

Initialize Trip

Do While (Stopping Criteria Not Satisfied) - Cycle Loop

Do Until (Each Ant Completes a Tour) - Tour Loop

Local Trip Update

End Do 
Analyze Tours

Global Trip Update

End Do

\section{NEW ALGORITHM AND IMPLEMENTATION}

A. Following are the steps involved in providing communication path between two mobile nodes

- Cluster will be created using WCA and leader is elected. Cluster leader will communicate to its members using broadcast method while the mobile node will communicate to its cluster leader through unicast [9]. By implementing broadcast method, cluster leader need not try to figure out the location of the mobile node. Thus reducing the computations required to locate the mobile node.

- A node who can listen to more than two cluster leader is identified as a Cluster Gateway (CG).

- Source node initiate communication to its cluster leader.

- Cluster leader of source node (SCL) will search for the cluster leader where the destination node registered (DCL) with the help of cluster gateway.

- The route discovery process between two cluster leaders and cluster gateway is done using ACO.

- Figure1 gives the flowchart of node to node communication at the cluster gateway once an ant is received.

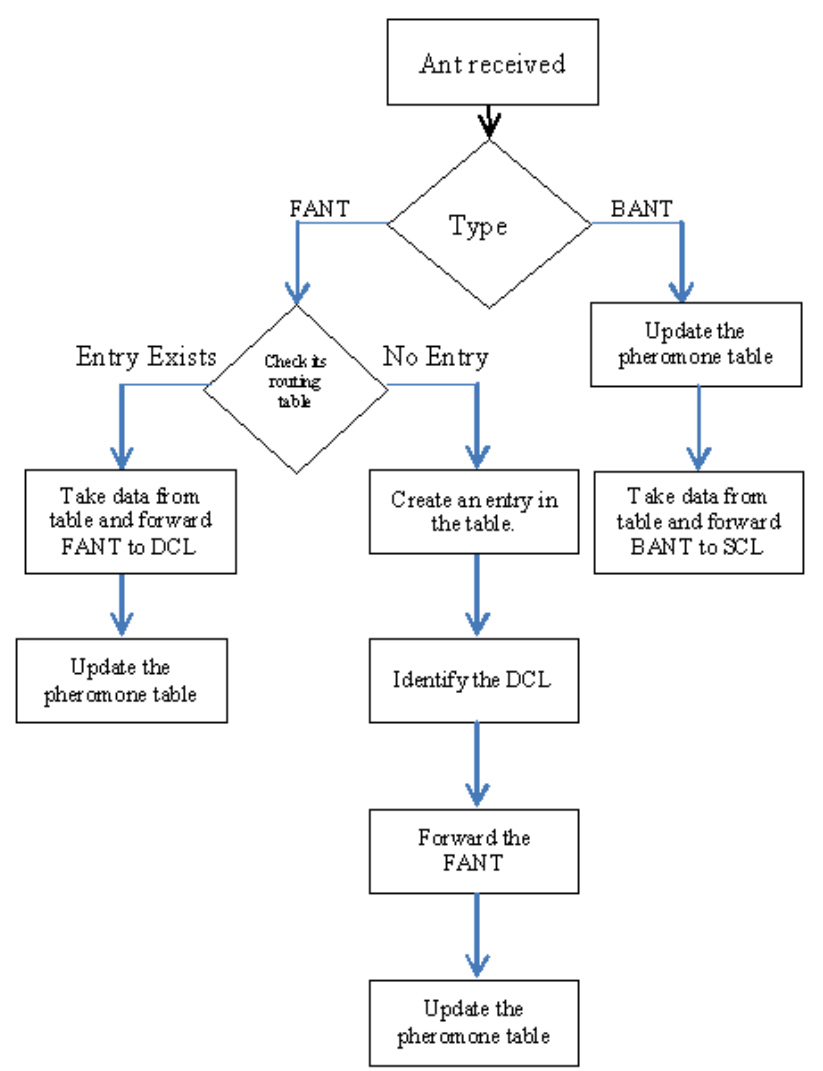

Figure1. Flowchart of node to node communication using ACO after creating cluster using WCA 
- After the path between the two cluster leaders been identified source node will send the data to its SCL.

- SCL will send the data to the CG.

- CG intern will forward the data to the DCL.

- Finally DCL will broadcast in its region so that the destination node will get the data easily.

B. Following are the steps involved to provide multicasting

- Cluster is created using WBCA and leader is elected.

- Cluster gateway is identified

- Cluster gateway finds the cluster leaders involved in multicasting.

- Multicast tree is constructed using ACO.

- Figure2 gives the flowchart of multicast communication at the cluster gateway once an ant is received.

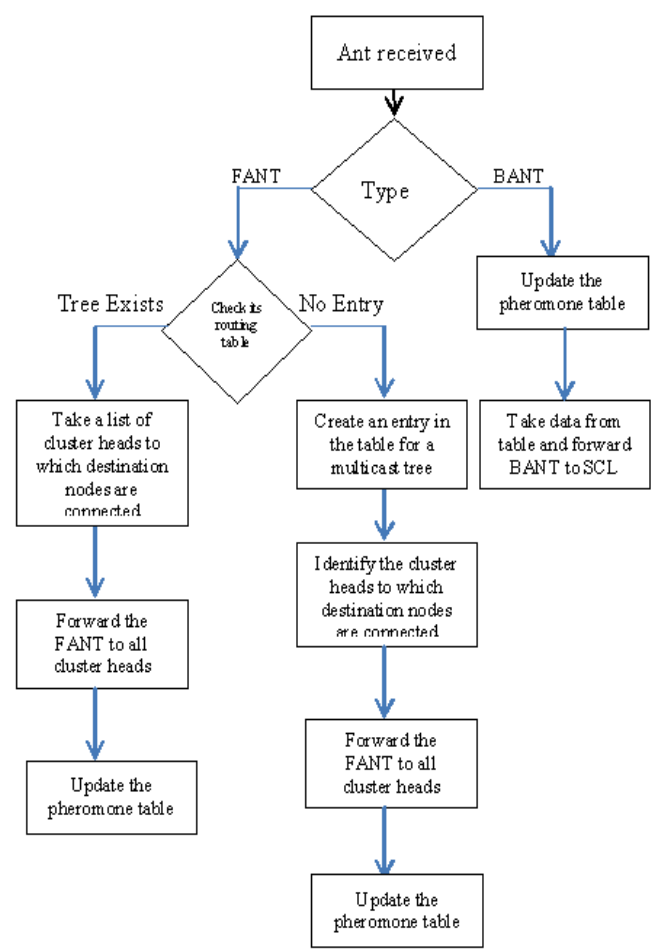

Figure2. Flowchart of Multicast communication using ACO after creating cluster using WCA

- After construction of multicast tree, source node will send the data to its cluster leader, SCL.

- SCL will forward the data to CG.

- CG will construct messages to the cluster leader in multicast tree.

- Cluster leader of multicast tree will intern broadcast in their region.

\section{IV . SIMULATION STUDY}

\section{A. Simulation Testing Environment}

In this section, the performance of the new algorithm is analyzed. Evaluation of the algorithm is done through simulation using ns 2 as a simulation tool. Here a network is considered to be having 20 nodes randomly located in a 500*500 region. The transmission range of each node is considered to be $250 \mathrm{~m}$. Nodes can move up to maximum speed of $5 \mathrm{~m} / \mathrm{s}$. Assumed channel capacity is $2 \mathrm{Mb} / \mathrm{s}$ and propagation model is free space model. Each simulation runs for 500 seconds. 


\section{B. Simulation Results}

Two scenarios of experiments have been conducted.

In Scenario1, performance of node to node communication using AODV and performance of node to node

Communication by creating clusters using WCA and implementing ACO over it. Here source and destination nodes are chosen randomly from 20 nodes. Simulation compares the throughput and Packet Delivery Ratio (PDR) of new algorithm and AODV. Figure 3 shows the simulation results which gives throughput. Figure 4 shows the simulation results which gives PDR.

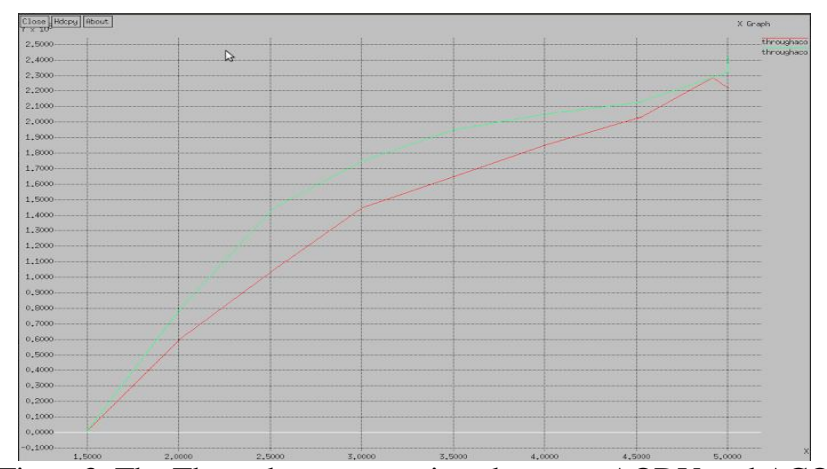

Figure3. The Throughput comparison between AODV and ACO

From figure 3, mechanism proposed by new algorithm ensures that the throughput is more compared to AODV and can be achieved in four hops.

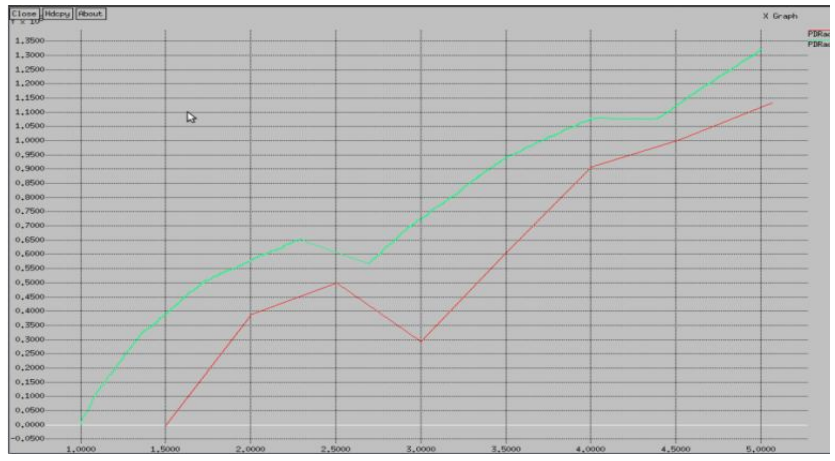

Figure4. Packet Delivery Ratio comparison between AODV and ACO

From the figure 4, it can be clearly analyzed that the new algorithm which implements ACO over WCA gives more PDR compared to AODV.

In Scenario2, performance of multicast communication using AODV, the performance of multicast communication by creating clusters using WCA and implementing ACO over it and the performance of the algorithm by implementing the AODV at every node that is willing to join multicast (One AODV) is simulated. Here source and destination nodes are chosen randomly from 20 nodes. Nodes who want to join multicast tree will communicate their cluster leaders and cluster leaders of those nodes will communicate to CG. CG will take the responsibility of creating multicast tree with the help of ACO. Simulation compares the Packet loss of AODV, new algorithm and One AODV. Figure 5 shows the simulation results obtained. 


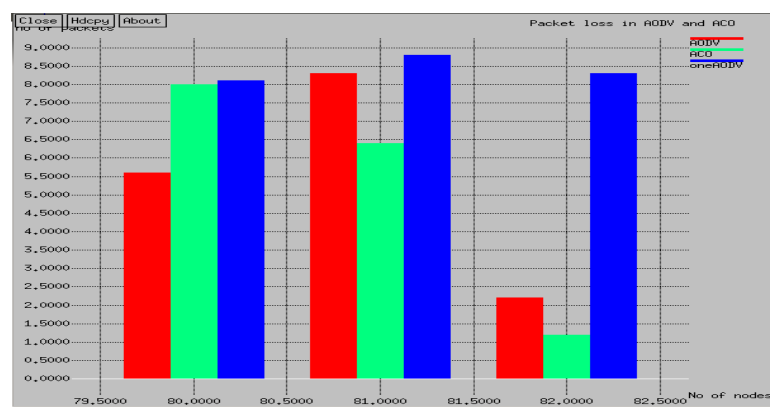

Figure5. Comparison of Packet loss in AODV and ACO

Figure 5 clearly shows that the packet loss decreases for ACO with clustering technique whereas Packet loss increases for AODV is more and the packet loss of One AODV is much more than the other two algorithms over a period of time.

\section{ACKNOWLEDGMENT}

The authors thank Central Computing Facility of R.V. College of Engineering, Bangalore, India and BMSCE management, Bangalore, India. The authors also thank Principal of R.V.College of Engineering and Principal of BMSCE, Bangalore, for the support given.

\section{CONCLUSION}

Clusters are created and cluster leaders are elected using WCA. After determining the cluster leaders, a cluster gateway is selected who can contact more than two cluster leader. Considering simulation performed on $\mathrm{ns} 2$ and analysis on obtained graphs, throughput obtained and the packet delivery ratio is significantly more compare to AODV. And to achieve multicast communication, packet loss is less with ACO compared to AODV and significantly less in One AODV.

From the above analysis, it could be concluded that the Ant Based Routing Algorithm over Weighted Clustering Algorithm can provide better unicast and multicast communication for MANETs.

\section{REFERENCES}

[1] Aarti, Dr. S. S. Tyagi," Study of MANET: Characteristics, Challenges, Application and Security Attacks", International Journal of Advanced Research in Computer Science and Software Engineering,Volume 3, Issue 5, May 2013

[2] Kong Ling Pang, Yang Qin, "The Comparison Study of Flat Routing and Hierarchical Routing in Ad Hoc Wireless Networks",2006 14th IEEE International Conference on Networks (Volume:1 ) Singapore, Sept. 2006, Page(s): 1 - 6, DOI:10.1109/ICON.2006.302584

[3] Tanmoy Kanti Halder, Chandreyee Chowdhury; Sarmistha Neogy, "Power Aware AODV Routing Protocol for MANET", Publisher: IEEE,27-29 Aug. 2014 Page(s):331 - 334, DOI: $10.1109 /$ ICACC. 2014.84

[4] Osamah S. Badarneh andMichel Kadoch,'Multicast Routing Protocols inMobile Ad Hoc Networks: A Comparative Survey and Taxonomy", Hindawi Publishing Corporation EURASIP Journal onWireless Communications and Networking Volume 2009, Article ID 764047, 42 pages doi:10.1155/2009/764047

[5] Abbas Karimi, Abbas Afsharfarnia,Faraneh Zarafshan, Al-Haddad, A Novel Clustering Algorithm for Mobile Ad Hoc Networks Based on Determination of Virtual Links' Weight to Increase Network Stability, ScientificWorldJournal. 2014; 2014: 432952.Published online 2014 Apr 30. DOI: $10.1155 / 2014 / 432952$

[6] Sowmya K S, N. K. Cauvery, "Weighted Clustering Algorithm with Ant ColonyOptimization to Provide Better Quality of Service", International Journal of Computer Science and Information Technologies, Vol. 5 (6) , 2014, 7702-7705,ISSN 0975-9646 
[7] Ramanjyot Kaur, "Swarm Intelligence based Routing Algorithms for MANETs: A Review",International Journal of Science Technology \& Engineering | Volume 2 | Issue 07 | January 2016, ISSN (online): 2349-784X

[8] O.A. Mohamed Jafar and R. Sivakumar, "Ant-based Clustering Algorithms: A Brief Survey “,International Journal of Computer Theory and Engineering, Vol. 2, No. 5, October, 2010 ,1793-8201

[9] A. Sabari, K.Duraiswamy, "Ant Based Adaptive Multicast Routing Protocol(AAMRP) for Mobile Ad Hoc Networks”, International Journal of Computer Science and Information Security,Vol.6, No. 2, 2009 